\title{
Atypical hemolytic uremic syndrome after childbirth: a case report
}

\author{
Hong Sang Choi ${ }^{1}$, Jae Won Yun ${ }^{2,3}$, Hee-Jin Kim ${ }^{2}$, Doyeun $\mathrm{Oh}^{4}$, Nah Ihm Kim ${ }^{5}$, Chang Seong Kim ${ }^{1}$, \\ Seong Kwon $\mathrm{Ma}^{1}$, Soo Wan Kim ${ }^{1}$, Eun Hui Bae ${ }^{1 \wedge}$ \\ ${ }^{1}$ Department of Internal Medicine, Chonnam National University Medical School, Gwangju, Korea; ${ }^{2}$ Department of Laboratory Medicine and \\ Genetics, Samsung Medical Center, Sungkyunkwan University School of Medicine, Seoul, Korea; ${ }^{3}$ Veterans Medical Research Institute, Veterans \\ Health Service Medical Center, Seoul, Korea; ${ }^{4}$ Department of Internal Medicine, School of Medicine, CHA University, School of Medicine, \\ Seongnam, Korea; ${ }^{5}$ Department of Pathology, Chonnam National University Medical School, Gwangju, Korea \\ Correspondence to: Eun Hui Bae, MD, PhD. Department of Internal Medicine, Chonnam National University Medical School, 42 Jebongro, Gwangju \\ 61469, Korea. Email: baedak76@gmail.com.
}

\begin{abstract}
We report a case of atypical hemolytic uremic syndrome (HUS) that occurred after childbirth. A 33-year-old female was admitted to the emergency room, complaining of abdominal pain six days after giving birth to twins. The patient was diagnosed with hemoperitoneum due to hepatic hemangioma rupture and a left lateral hepatectomy was performed. Angioembolization was performed for the accompanying uterine artery bleeding. After that, her kidney function worsened after the 12th day postpartum. Microangiopathic anemia, thrombocytopenia and renal dysfunction were observed. Shiga toxin-producing Escherichia coli was negative in the stool. Plasma ADMATS 13 activity was normal. After transfer to the nephrology department with suspected atypical HUS, the patient underwent fresh frozen plasma (FFP) transfusion with three hemodialysis sessions. The patient improved without additional dialysis, but a renal biopsy was performed because of persistent proteinuria. Renal pathologic findings were compatible with thrombotic microangiopathy. A genetic test for atypical HUS revealed variants of uncertain significance in the complement factor $\mathrm{H}$ related (CFHR) 4 gene and the presence of CFHR3-CFHR1 copy number gain. The CFHR3-CFHR1 copy number gain found in this case is a rare causative mutation of atypical HUS. This case suggests that genetic testing of atypical HUS should include analysis of $C F H$-CFHR rearrangements as well as general screening for complement-associated genes.
\end{abstract}

Keywords: Atypical hemolytic uremic syndrome (HUS); CFHR3-CFHR1; copy number gain; pregnancy; case report

Submitted May 08, 2020. Accepted for publication Sep 30, 2020.

doi: $10.21037 / \mathrm{atm}-20-3789$

View this article at: http://dx.doi.org/10.21037/atm-20-3789

\section{Introduction}

Hemolytic uremic syndrome (HUS) is a comparatively rare hematological abnormality that accompanies microangiopathic hemolytic anemia (MAHA), thrombocytopenia, and acute kidney injury (AKI). Atypical HUS is caused by inborn or acquired genetic defects of genes that are associated with regulation of the complement pathway. Atypical HUS is known to be triggered by factors that induce the activation of complement cascades, including infection, pregnancy, organ transplantation, malignancy, autoimmune disease, and drugs (1). Cases of pregnancyassociated atypical HUS are known to occur during the postpartum period (2). More than half of thrombotic microangiopathies associated with pregnancy have genetic abnormalities in the complement system. Abnormalities of several genes associated with the complement system have been reported (3), and mutations can be diagnosed relatively easily using advanced diagnostic methods, such

^ ORCID: 0000-0003-1727-2822. 


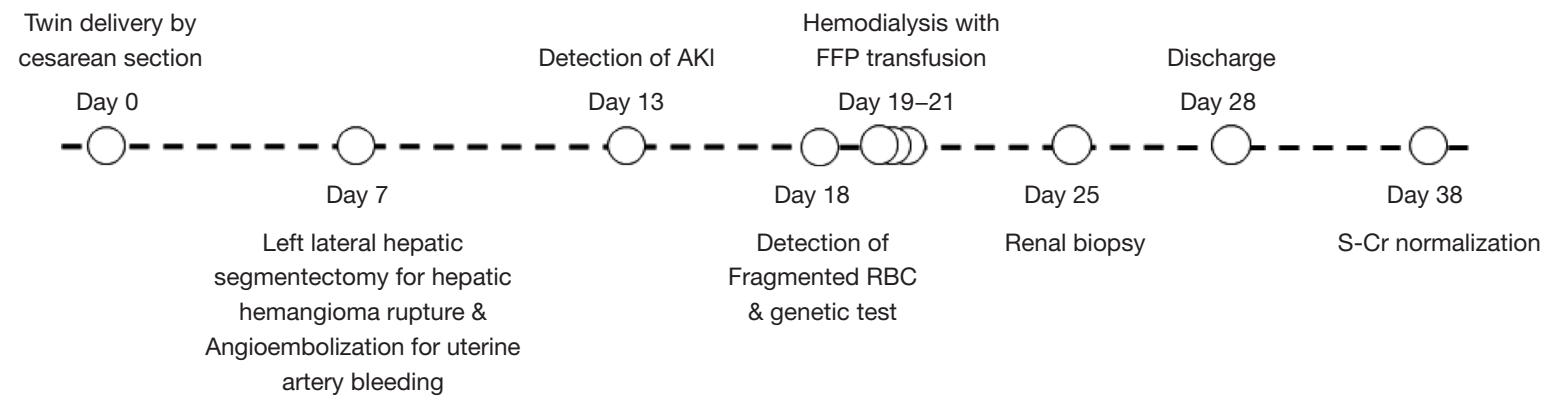

Figure 1 Timeline of patient's clinical course. AKI, acute kidney injury; RBC, red blood cell; FFP, fresh frozen plasma; s-Cr, serum creatinine.

as new generation sequencing (NGS). Similarly, copy number variation resulting from genomic rearrangement can be diagnosed using techniques like multiplex ligationdependent probe amplification (MLPA) (4). Here, we report successful treatment of a patient who was diagnosed with postpartum atypical HUS after childbirth by identification of genetic abnormalities in the complement system using NGS and MLPA. This case is an atypical HUS due to the rare copy number variation of the CFHR genes, and shows that the genetic test for atypical HUS must be performed for CFH-CFHR rearrangement. We present the following article in accordance with the CARE reporting checklist (available at http://dx.doi.org/10.21037/atm-20-3789) (5).

\section{Case presentation}

A 33-year-old woman with renal impairment was referred to our nephrology department. She had delivered twins through Caesarean section 18 days before referral. She denied any underlying disease, medication history or familial history of renal impairment. Six days after delivery, she had received left lateral hepatic segmentectomy and angioembolization of the uterine artery due to hepatic hemangioma rupture and uterine artery bleeding. Her serum creatinine level started to increase approximately 12 days after delivery and continued to increase despite proper management (Figure 1). On the day of referral, her laboratory data were: white blood cell count, $8,800 / \mu \mathrm{L}$; hemoglobin, $7.7 \mathrm{~g} / \mathrm{dL}$; platelet count, $68,000 / \mu \mathrm{L}$; blood urea nitrogen, $33.2 \mathrm{mg} / \mathrm{dL}$; serum creatinine, $2.46 \mathrm{mg} / \mathrm{dL}$; and lactate dehydrogenase (LDH), 3,958 U/L. Peripheral blood smears were performed for further evaluation of bicytopenia and showed fragmented red blood cells. Together, these results were indicative of thrombotic microangiopathy. The patient had no recent history of diarrhea and no enterohemorrhagic Escherichia coli was detected in her stool. Plasma ADMATS 13 activity was $60 \%$. The lack of accompanying neurological symptoms led us to suspect atypical HUS. Renal biopsy revealed findings consistent with thrombotic microangiopathy (Figure 2).

Hemodialysis was initiated immediately and the patient was administered fresh frozen plasma (FFP). FFP was administered with three sessions of dialysis, after which her laboratory findings began to improve. Platelet counts improved without blood transfusion and serum LDH levels gradually decreased. This trend persisted even after cessation of dialysis and FFP administration. The patient was discharged 10 days after being transferred to the department of nephrology. No signs of renal failure or recurrence of atypical HUS were apparent on her fourmonth outpatient follow-up, at which her laboratory findings were: serum creatinine level, $0.53 \mathrm{mg} / \mathrm{dL}$; platelet count of $219,000 / \mu \mathrm{L}$; and serum LDH level, $378 \mathrm{U} / \mathrm{L}$.

Genetic testing was performed concurrently with the treatment regime to identify the cause of the atypical HUS. Sequencing of 17 genes known to be associated with atypical HUS (i.e., C3, C4BPA, CD46, CFB, CFH, CFHR1, CFHR2, CFHR3, CFHR4, CFHR 5, CFI, DGKE, THBD, $P L G$, etc.) as well as of $A D A M T S 13$ and $C 5$ was performed. Gene sequencing was performed by Illumina Hi-seq 2500 platform, followed by targeted Sanger sequencing for validation of identified genetic variants. Gene dosage tests were performed using MLPA. Genetic profiling revealed a substitutional variant c. $1607 \mathrm{~T}>\mathrm{G}$ \{p.[Ile536Arg] or I536R in CFHR4. Additionally, MLPA analysis revealed the presence of a copy number gain in the CFHR3-CFHR1 regions (Figure 3). Genetic testing confirmed a diagnosis of atypical HUS caused by a point mutation and copy number gain in CFHR. About 1 year after the onset of the disease, her serum creatinine level was $0.55 \mathrm{mg} / \mathrm{dL}$, and there was 


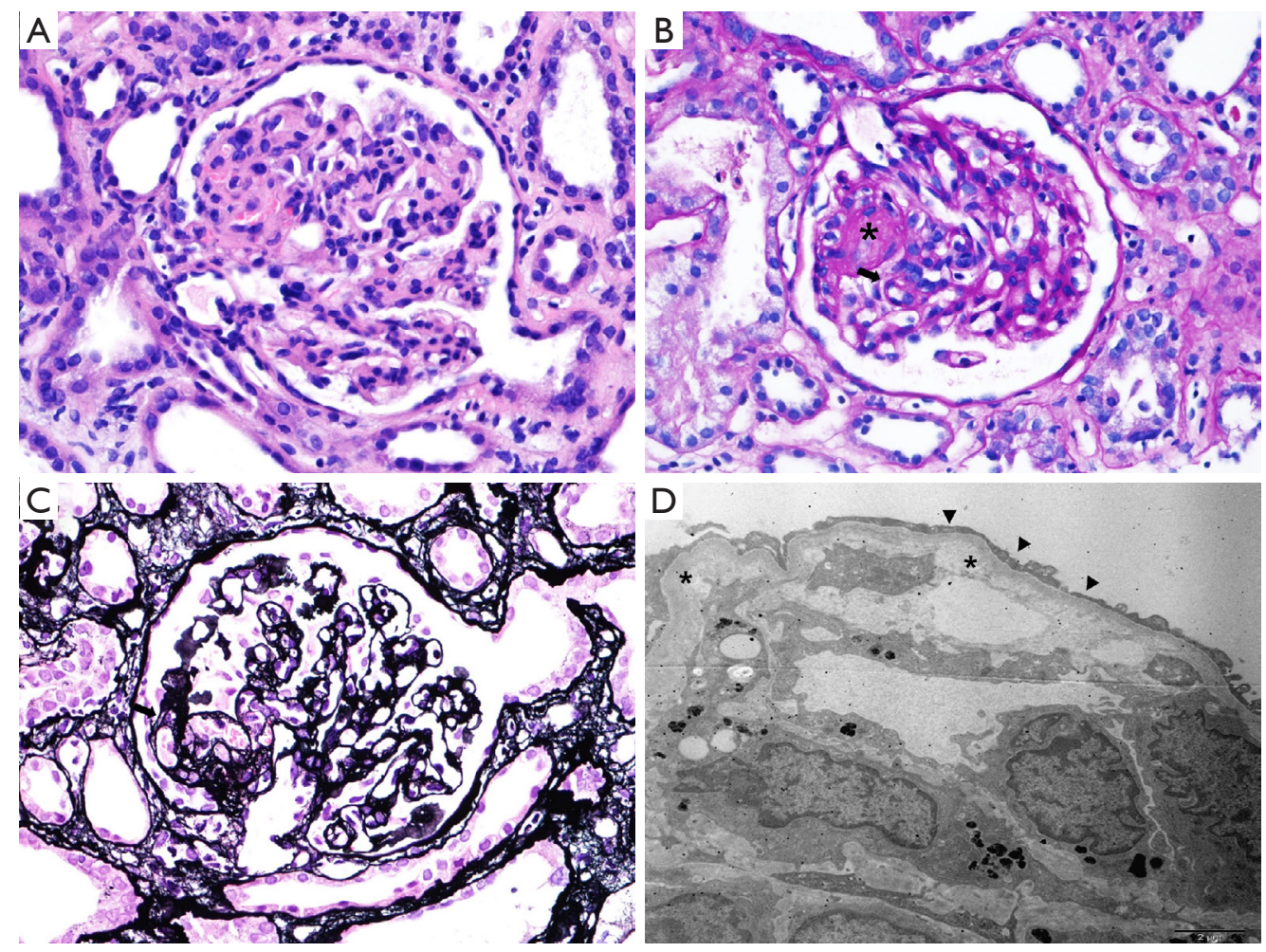

Figure 2 Renal biopsy. (A) Glomeruli show thickened capillary wall and fragmented red blood cells are trapped in the mesangial matrix (hematoxylin and eosin stain, $\times 40$ ). (B) The periodic acid Schiff (PAS) stained glomerulus demonstrates segmental mesangiolysis (asterisk) and double contours in capillary basement membrane (arrow) $(\times 40)$. (C) Light microscopy image of the kidney biopsy specimen shows double contours of the glomerular basement membrane with Jones methenamine silver stain ( $\times 40)$. (D) Electron microscopy of the kidney biopsy reveals irregular electron-lucent expansion of the subendothelial zone (asterisk) and diffuse foot process fusion (arrowhead) $(\times 8,000)$.

no recurrence or unanticipated outcome during the followup period.

All procedures performed in studies involving human participants were in accordance with the ethical standards of the institutional and/or national research committee(s) and with the Helsinki Declaration (as revised in 2013). Written informed consent was obtained from the patient.

\section{Discussion}

We present a case of pregnancy-associated atypical HUS during the postpartum period in a young woman who has two genetic mutations in the complement cascade. The patient had previously given birth and had no history of diagnosis or treatment with thrombocytopenia or AKI. Fakhouri et al. reported that approximately $20 \%$ of atypical HUS patients in France are related to pregnancy; 80\% of these were reported during the postpartum period, with the time of onset varying widely from the $3^{\text {rd }}$ day postpartum to the $4^{\text {th }}$ month (2). The development of HUS in the current case occurred 12 days postpartum, consistent with previous reports. Initially, development of HUS can be detected through a decrease in platelet counts and AKI. Differentiation from other causes of AKI that may occur during pregnancy, including preeclampsia, eclampsia, HELLP (hemolysis, elevated liver enzymes, low platelet) syndrome, and thrombotic thrombocytopenic purpura (TTP), is important (6). Other common causes of AKI, such as volume depletion or acute tubular necrosis, should also be considered. In particular, TTP is very similar to the expression of HUS, which can complicate differential diagnosis. However, even before ADAMTS13 activity test results are confirmed, TTP is more thrombocytopenic and has less severe renal failure than HUS. Furthermore, TTP predominantly occurs in the $2^{\text {nd }}$ or $3^{\text {rd }}$ trimester of pregnancy (7).

Genomic rearrangement in the $C F H-C F H R$ region resulting in copy number gain of $C F H R 3$ and CFHR 1 

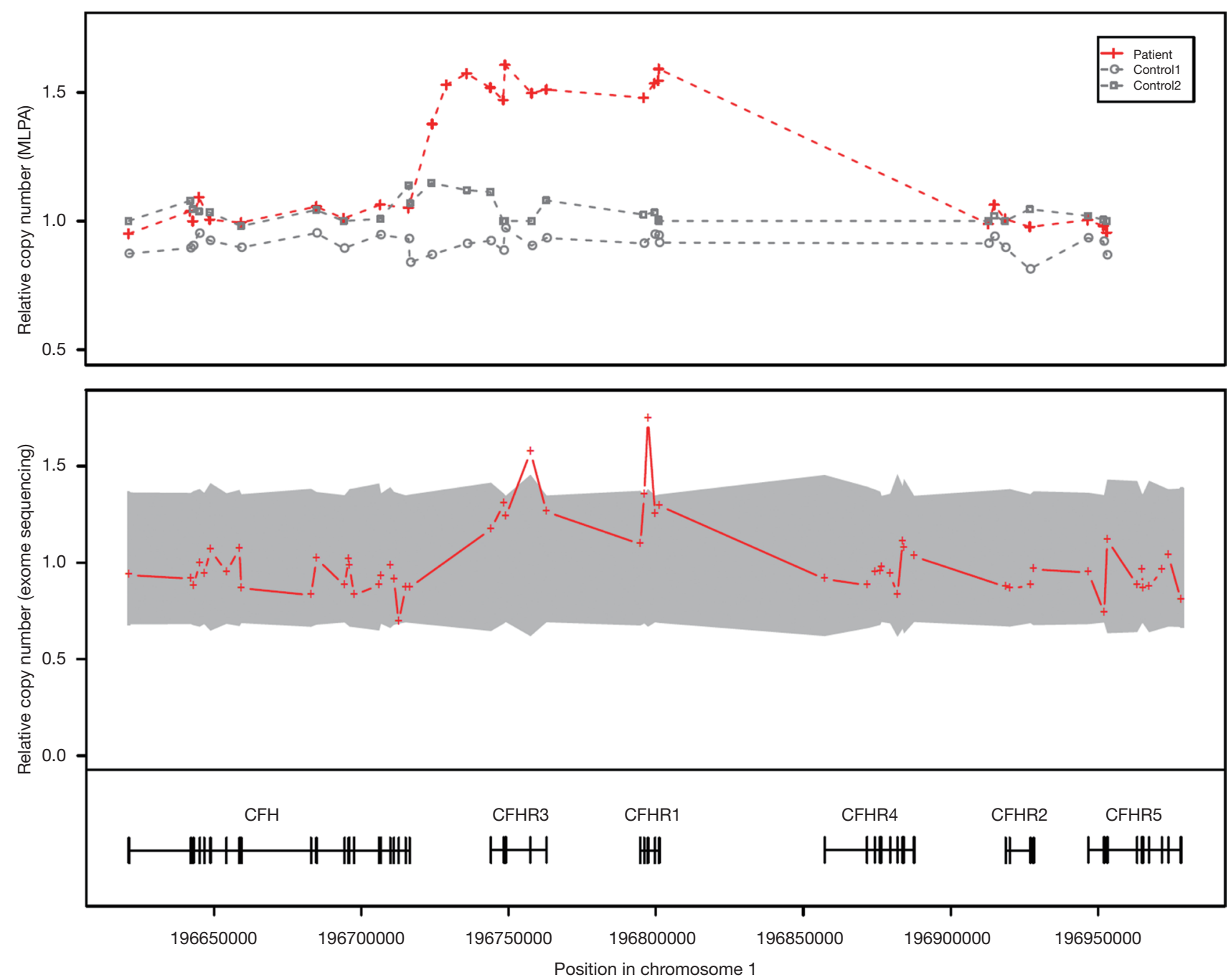

Figure 3 The relative copy number based on the multiplex ligation-dependent probe amplification (MLPA) study (upper plot). The value 1.0 on Y axis represents two copies or copy neutral. Each “+” represents each probe in MLPA study. The relative copy number based on the exome sequencing copy number analysis (lower plot). Each “+” represents an exon region in exome sequencing study. The grey zone indicates $99 \%$ confidence interval of normal controls without copy number alterations.

was discovered in our patient by MLPA analysis. Several previous studies have reported atypical HUS by the CFHR1/CFH hybrid gene $(4,8,9)$. Valoti et al. reported the generation of $C F H R 1 / C F H$ hybrid gene and an extra copy of CFHR3 and CFHR1 in a patient with atypical HUS (4). Although the patient in the current case did not have a CFHR1/CFH hybrid gene, her atypical HUS was likely caused by a CFHR3-CFHR1 copy number gain, which is similar to a variant described by Valoti et al. (4). In addition to the copy number gain of CFHR3-CFHR1, a variant of uncertain significance was found in the CFHR4 gene in our patient. The function of CFHR4 remains unclear, though it is known to bind to the $\mathrm{C} 3 \mathrm{~b}$ and to have a complement modulatory activity in the form of a factor $\mathrm{H}$ cofactor enhancing activity (10). The I536R mutation in our patients is located in CFHR4 SCR9, and its role in protein function and disease association is unknown. In addition, except for a deletion of CFHR1/CFHR4, no other CFHR4 mutation has been reported to be associated with atypical HUS.

Our study found a very rare CFHR3-CFHR1 copy number gain as the cause of atypical HUS. However, our study has some limitations. First, a functional study was 
not performed on the discovered mutations. In addition, the mechanism of how CFHR3-CFHR1 copy number gain generated atypical HUS could not be revealed. Second, although no family member with similar symptoms was found, it is a limitation of our study that genetic testing of the family members was not performed because of that.

In summary, we describe a case of atypical HUS that occurred during the postpartum period and was likely caused by a CFHR3-CFHR1 copy number gain. This case suggests that genetic testing of atypical HUS should include analysis of $C F H-C F H R$ rearrangements as well as general screening for complement-associated genes.

\section{Acknowledgments}

Funding: This study was supported by the Korean Ministry of Education (2017R1D1A1B03029582) and grant (BCRI20076 and BCRI20041) of Chonnam National University Hospital Biomedical Research Institute.

\section{Footnote}

Reporting Checklist: The authors have completed the CARE reporting checklist. Available at http://dx.doi.org/10.21037/ atm-20-3789

Conflicts of Interest: All authors have completed the ICMJE uniform disclosure form (available at http://dx.doi. org/10.21037/atm-20-3789). The authors have no conflicts of interest to declare.

Ethical Statement: The authors are accountable for all aspects of the work in ensuring that questions related to the accuracy or integrity of any part of the work are appropriately investigated and resolved. All procedures performed in studies involving human participants were in accordance with the ethical standards of the institutional and/or national research committee(s) and with the Helsinki Declaration (as revised in 2013). Written informed consent was obtained from the patient for publication of this manuscript and any accompanying images.

Open Access Statement: This is an Open Access article distributed in accordance with the Creative Commons Attribution-NonCommercial-NoDerivs 4.0 International License (CC BY-NC-ND 4.0), which permits the noncommercial replication and distribution of the article with the strict proviso that no changes or edits are made and the original work is properly cited (including links to both the formal publication through the relevant DOI and the license). See: https://creativecommons.org/licenses/by-nc-nd/4.0/.

\section{References}

1. Lee H, Kang E, Kang HG, et al. Consensus regarding diagnosis and management of atypical hemolytic uremic syndrome. Korean J Intern Med 2020;35:25-40.

2. Fakhouri F, Roumenina L, Provot F, et al. Pregnancyassociated hemolytic uremic syndrome revisited in the era of complement gene mutations. J Am Soc Nephrol 2010;21:859-67.

3. Noris M, Bresin E, Mele C, et al. Genetic Atypical Hemolytic-Uremic Syndrome. In: Adam MP, Ardinger HH, Pagon RA, et al. editors. GeneReviews(®). Seattle (WA); 1993.

4. Valoti E, Alberti M, Tortajada A, et al. A novel atypical hemolytic uremic syndrome-associated hybrid CFHR1/ $\mathrm{CFH}$ gene encoding a fusion protein that antagonizes factor H-dependent complement regulation. J Am Soc Nephrol 2015;26:209-19.

5. Gagnier JJ, Kienle G, Altman DG, et al. The CARE Guidelines: Consensus-based Clinical Case Reporting Guideline Development. Glob Adv Health Med 2013;2:38-43.

6. Baghli S, Abendroth C, Farooq U, et al. Atypical Presentation of Pregnancy-Related Hemolytic Uremic Syndrome. Am J Kidney Dis 2018;72:451-6.

7. Fakhouri F. Pregnancy-related thrombotic microangiopathies: Clues from complement biology. Transfus Apher Sci 2016;54:199-202.

8. Alobaidi S, AlDabbagh A, Alamoudi A, et al. Three months interval therapy of Eculizumab in a patient with atypical hemolytic uremic syndrome with hybrid CFHR1/ CFH gene. CEN Case Rep 2019;8:139-43.

9. Eyler SJ, Meyer NC, Zhang Y, et al. A novel hybrid CFHR1/CFH gene causes atypical hemolytic uremic syndrome. Pediatr Nephrol 2013;28:2221-5.

10. Skerka C, Chen Q, Fremeaux-Bacchi V, et al. Complement factor $\mathrm{H}$ related proteins (CFHRs). Mol Immunol 2013;56:170-80.

Cite this article as: Choi HS, Yun JW, Kim HJ, Oh D, Kim NI, Kim CS, Ma SK, Kim SW, Bae EH. Atypical hemolytic uremic syndrome after childbirth: a case report. Ann Transl Med 2021;9(1):79. doi: 10.21037/atm-20-3789 